\section{Comment on "Minimum Action Path Theory Reveals the Details of Stochastic Transitions Out of Oscillatory States"}

In a recent Letter [1], de la Cruz et al. studied a noiseinduced transition in an oscillating stochastic population undergoing birth- and death-type reactions. When described by deterministic rate equations, the population approaches a stable limit cycle. The intrinsic noise, caused by the discreteness of molecules and randomness of their interactions, leads to an escape from this limit cycle through an adjacent unstable limit cycle, and de la Cruz et al. attempted to evaluate the mean first passage time (MFPT) to escape.

A crucial approximation, made in the Letter, was to replace the original master equation by the "chemical Langevin equation" (CLE), their Eq. (2). Unfortunately, this standard procedure, based on the van Kampen expansion in the inverse population size $1 / \Omega \ll 1$ [2], applies only for typical, small fluctuations around the stable limit cycle. It fails in the tails of the metastable quasistationary distribution of the population size around the limit cycle. One of these tails determines the escape rate of the population through the unstable limit cycle. As a result, the MFPT, predicted by de la Cruz et al., involves an error that grows exponentially with the population size $\Omega \gg 1$, due to an error in the calculation of $\mathcal{S}$. In this situation, their study of a preexponential factor in the MFPT does not have much meaning.

The inadequacy of the van Kampen system-size expansions for a description of large fluctuations in Markov jump processes is, by now, well documented [3-9]. The only general exception appears when the system is close to the proper bifurcation of the underlying deterministic model [5,9-12]. In the present case, it is the saddle-node bifurcation of the stable and unstable limit cycles.

Fortunately, there is no need for uncontrolled approximations. The Freidlin-Wentzell Wentzel-KramersBrillouin (WKB) theory was extended to stochastic populations quite some time ago [13-15]. The corresponding WKB technique employs the same large parameter $\Omega \gg 1$ but circumvents the van Kampen system-size expansion, see, e.g., Ref. [9] for a recent review. Moreover, this WKB technique was already applied to escape from a limit cycle, in the context of the extinction of long-lived oscillating populations [16].

Even within the framework of the CLE, much of the Letter is devoted to a rediscovery of known results, as de la Cruz et al. seem to be unaware of a body of important

Published by the American Physical Society under the terms of the Creative Commons Attribution 4.0 International license. Further distribution of this work must maintain attribution to the author(s) and the published article's title, journal citation, and DOI. previous analytical, numerical, and experimental works on the noise-induced escape from limit cycles and from attractors of dynamical systems in general [17-22]. A proper formulation of the Freidlin-Wentzell escape optimization problem, which was put forward in these works, and which is lacking in the Letter, involves the time interval $-\infty<t<\infty$. A minimum action path-an instantonexits the limit cycle at $t=-\infty$ while performing an infinite number of loops. There is a whole one-parameter family of instanton solutions, linked to one another through the time translations $t \rightarrow t+$ const, and each instanton yields the same classical action. Any evidence to the contrary results from finite-time numerical artifacts.

We acknowledge discussions with M. I. Dykman and support from the Israel Science Foundation (Grant No. 807/16). N. R. S. was supported by the Clore Foundation.

\section{Baruch Meerson ${ }^{*}$ and Naftali R. Smith ${ }^{\dagger}$ \\ Racah Institute of Physics \\ Hebrew University of Jerusalem \\ Jerusalem 91904, Israel}

Received 2 April 2018; published 8 February 2019

DOI: 10.1103/PhysRevLett.122.059801

*meerson@mail.huji.ac.il †naftalismith@gmail.com

[1] R. de la Cruz, R. Perez-Carrasco, P. Guerrero, T. Alarcon, and K. M. Page, Phys. Rev. Lett. 120, 128102 (2018).

[2] N. van Kampen, Stochastic Processes in Physics and Chemistry (Elsevier, New York, 2007).

[3] B. Gaveau, M. Moreau, and J. Toth, Lett. Math. Phys. 37, 285 (1996).

[4] V. Elgart and A. Kamenev, Phys. Rev. E 70, 041106 (2004).

[5] C. R. Doering, K. V. Sargsyan, and L. M. Sander, Multiscale Model. Simul. 3, 283 (2005).

[6] D. A. Kessler and N. Shnerb, J. Stat. Phys. 127, 861 (2007).

[7] M. Assaf and B. Meerson, Phys. Rev. E 75, 031122 (2007).

[8] O. Ovaskainen and B. Meerson, Trends Ecol. Evol. 25, 643 (2010).

[9] M. Assaf and B. Meerson, J. Phys. A 50, 263001 (2017).

[10] B. Meerson and P. V. Sasorov, Phys. Rev. E 78, 060103(R) (2008).

[11] C. Escudero and A. Kamenev, Phys. Rev. E 79, 041149 (2009).

[12] M. Assaf and B. Meerson, Phys. Rev. E 81, 021116 (2010).

[13] R. Kubo, K. Matsuo, and K. Kitahara, J. Stat. Phys. 9, 51 (1973).

[14] H. Gang, Phys. Rev. A 36, 5782 (1987).

[15] M. I. Dykman, E. Mori, J. Ross, and P. M. Hunt, J. Chem. Phys. 100, 5735 (1994).

[16] N. R. Smith and B. Meerson, Phys. Rev. E 93, 032109 (2016).

[17] R. L. Kautz, Phys. Lett. A 125, 315 (1987). 
[18] R. L. Kautz, Phys. Rev. A 38, 2066 (1988).

[19] P. Grassberger, J. Phys. A 22, 3283 (1989).

[20] R. L. Kautz, Rep. Prog. Phys. 59, 935 (1996).

[21] V. N. Smelyanskiy, M. I. Dykman, and R. S. Maier, Phys. Rev. E 55, 2369 (1997); 56, 2332 (1997).
[22] I. A. Khovanov, D. G. Luchinsky, R. Mannella, and P. V. E. McClintock, in Stochastic Processes in Physics, Chemistry and Biology, edited by J.A. Freund and T. Pöschel (Springer, Berlin, 2000), p. 378. 